Pacific

Journal of

Mathematics

ON THE CONVEXITY OF THE KOBAYASHI METRIC ON A TAUT COMPLEX MANIFOLD

Masashi KobaYashi

Volume 194 No. 1

May 2000 


\section{ON THE CONVEXITY OF THE KOBAYASHI METRIC ON A TAUT COMPLEX MANIFOLD}

\section{MASASHI KOBAYASHI}

We study the Kobayashi-Royden metric and the Kobayashi distance on a taut complex manifold. We prove that the derivative of the Kobayashi distance is equal to the KobayashiBusemann metric. This gives us the necessary and sufficient condition of the convexity of the Kobayashi-Royden metric.

\section{Introduction.}

Let $M$ be an $m$-dimensional complex manifold. We recall the definition of the Kobayashi-Royden pseudo-metric on $M$ :

$$
F_{M}(\xi)=\inf \left\{t>\left.0\right|^{\exists} f \in \mathcal{O}(\Delta, M) \text { such that } t f_{*}\left(d /\left.d \zeta\right|_{\zeta=0}\right)=\xi\right\},
$$

where $\xi \in \boldsymbol{T}_{p} M$ is a holomorphic tangent vector, $\Delta=\{\zeta \in \mathbb{C}|| \zeta \mid<1\}$, and $\mathcal{O}(\Delta, M)=\{f: \Delta \rightarrow M \mid f$ is a holomorphic mapping $\}$. Then, $F_{M}$ has the following properties:

(i) $F_{M}(\xi) \geq 0$ for any $\xi \in \boldsymbol{T}_{p} M$;

(ii) $F_{M}(\lambda \xi)=|\lambda| F_{M}(\xi)$ for any $\lambda \in \mathbb{C}$;

(iii) $F_{M}$ is upper semi-continuous on the holomorphic tangent bundle $\boldsymbol{T}_{p} M$, moreover if $M$ is taut, that is, $\mathcal{O}(\Delta, M)$ is a normal family;

(iv) $F_{M}$ is continuous on $\boldsymbol{T} M$;

(v) $F_{M}(\xi)=0$ if and only if $\xi=0$.

Hence we see that $F_{M}$ is a metric on $M$, if $M$ is taut.

Let $v \in T_{p} M$ be a real tangent vector. We can uniquely write $v=\xi+\bar{\xi}$ with $\xi \in \boldsymbol{T}_{p} M$. We set $F_{M}(v)=2 F_{M}(\xi)$. Then $F_{M}$ induce a pseudodistance $d_{M}$ on $M$ as follows:

$$
d_{M}(p, q)=\inf _{c}\left\{\int_{0}^{1} F_{M}\left(c_{*}(d / d t)\right) d t\right\}
$$

where $c$ runs over all piecewise smooth curves connecting $p$ with $q$. This pseudo-distance $d_{M}$ is called the integrated form of $F_{M}$. Since the condition $d_{M}(p, q)=0$ does not necessarily mean $p=q$ in general, $d_{M}$ is not a distance. We remark, however, that $d_{M}$ is a distance if $M$ is taut (see [7]).

We define the indicatrix of $F_{M}$ at $p$ by

$$
I_{F_{M}}(p)=\left\{\xi \in \boldsymbol{T}_{p} M \mid F_{M}(\xi)<1\right\} .
$$


Note that $F_{M}$ is a seminorm at $p$ if and only if its indicatrix at $p$ is a convex set. Hence we say that $F_{M}$ is convex at $p$, if it is a seminorm at $p$.

S. Kobayashi introduced a new infinitesimal pseudo-metric $\hat{F}_{M}$ on $M$ which is the double dual of $F_{M}$ (see [3]). It is defined by

$$
\hat{F}_{M}(\xi)=\inf \left\{t>0 \mid t^{-1} \xi \in \hat{I}_{F_{M}}(p)\right\}
$$

for all $\xi \in \boldsymbol{T}_{p} M$, where $\hat{I}_{F_{M}}(p)$ is the convex hull of $I_{F_{M}}(p)$. We call $\hat{F}_{M}$ the Kobayashi-Busemann pseudo-metric on $M . \hat{F}_{M}$ is a seminorm at any $p \in M$ and $\hat{F}_{M}$ is upper semi-continuous. Moreover $\hat{F}_{M}$ is a metric if $M$ is taut. Because $\hat{F}_{M}$ is a norm at any $p \in M$ and continuous on $\boldsymbol{T} M$, if $F_{M}$ is so. We also set $\hat{F}_{M}(v)=2 \hat{F}_{M}(\xi)$, where $\xi \in \boldsymbol{T}_{p} M$ with $v=\xi+\bar{\xi}$. Thus the integrated form of $\hat{F}_{M}$ are defined in the same way. S. Kobayashi in [3] proved that the integrated form of $\hat{F}_{M}$ is equal to that of $F_{M}$.

The integrated form of $F_{M}$ is, as a matter of fact, equal to the Kobayashi pseudo-distance (see [7]). It was originally defined as follows. First we define the function $d_{M}^{*}$ on $M \times M$ by

$$
\begin{aligned}
d_{M}^{*}(p, q)=\inf \left\{\left.\rho(a, b)\right|^{\exists} f \in \mathcal{O}(\Delta, M)\right. & \\
& \text { such that } a, b \in \Delta, f(a)=p \text { and } f(b)=q\},
\end{aligned}
$$

where $\rho$ is the Poincaré distance on $\Delta$. We set $d_{M}^{*}(p, q)=\infty$ if there is no analytic disc connecting $p$ with $q$. Note that $d_{M}^{*}(p, q)<\infty$ if $p$ is sufficiently close to $q$. Next for each positive integer $l$, we introduce the following function on $M \times M$ :

$$
d_{M}^{(l)}(p, q)=\inf \left\{\sum_{j=1}^{l} d_{M}^{*}\left(p_{j}, p_{j+1}\right) \mid p_{1}=p, p_{2}, \ldots, p_{l}, p_{l+1}=q \in M\right\} .
$$

Now we have the Kobayashi pseudo-distance on $M$ :

$$
d_{M}(p, q)=\lim _{l \rightarrow \infty} d_{M}^{(l)}(p, q) .
$$

By definition we easily see that

$$
d_{M}^{*}(p, q) \geq d_{M}^{(2)}(p, q) \geq \cdots \geq d_{M}^{(l)}(p, q) \geq \cdots \geq d_{M}(p, q)
$$

for all $p, q \in M$. We remark that $d_{M}^{*}(p, q)=d_{M}(p, q)$ if $M$ is a convex domain (see $[4]$ ).

Let $h$ be a Hermitian metric on $M$. We fix a point $p$ of $M$. Then, $h$ induces the exponential mapping exp: $U \rightarrow M$ where $U \subset T M$ is a small open neighborhood of $0 \in T_{p} M$. We define the derivative $\mathcal{D} d_{M}$ by

$$
\mathcal{D} d_{M}(v)=\lim _{\substack{u \rightarrow v \\ t \rightarrow 0}} \frac{d_{M}(q, \exp t u)}{|t|}
$$


where $u \in T_{q} M$, if the limit exists. We remark that this definition is independent of the choice of $h$. We set $\mathcal{D} d_{M}(\xi)=2^{-1} \mathcal{D} d_{M}(v)$ for $\xi \in \boldsymbol{T}_{p} M$, where $v=\xi+\bar{\xi}$. Then $\mathcal{D} d_{M}$ is a pseudo-metric on $M$.

Suppose $D$ is a domain in $\mathbb{C}^{m}$ with the standard flat metric. We identify $\boldsymbol{T} D$ with $D \times \mathbb{C}^{m}$. Then, if the derivative $\mathcal{D} d_{D}$ exists, we have

$$
\mathcal{D} d_{D}(p, \xi)=\lim _{\substack{(q, \eta) \rightarrow(p, \xi) \\ t \rightarrow 0}} \frac{d_{D}(q, q+t \eta)}{2|t|} .
$$

The derivative of the Kobayashi metric pseudo-distance on $M$ does not always exist. We know, however, the following facts: If $D$ is a domain in $\mathbb{C}^{m}$,

$$
\limsup _{\substack{(q, \eta) \rightarrow(p, \xi) \\ t \rightarrow 0}} \frac{d_{M}(q, q+t \eta)}{2|t|} \leq F_{D}(p, \xi)
$$

for each $(p, v) \in D \times \mathbb{C}^{m}$ (see [1]). On the other hand, M.Y. Pang showed in [6] that

$$
2 F_{D}(p, \xi)=\lim _{t \rightarrow 0} \frac{d_{D}^{*}(p, p+t \xi)}{|t|}
$$

for each $\xi \in \mathbb{C}^{m}$ when $D$ is a taut domain in $\mathbb{C}^{m}$.

Here we state our main theorem about the derivative of the Kobayashi distance.

Main Theorem 1.1. If $M$ is a taut complex manifold, then $\mathcal{D} d_{M}$ exists and

$$
\mathcal{D} d_{M}=\hat{F}_{M} .
$$

This theorem gives us the following formula:

$$
\lim _{\substack{(q, \eta) \rightarrow(p, \xi) \\ t \rightarrow 0}} \frac{d_{D}(q, q+t \eta)}{|t|}=2 \hat{F}_{D}(p, \xi)
$$

for each $\xi \in \mathbb{C}^{m}$, if $D$ is a taut domain in $\mathbb{C}^{m}$. Considering the result of the derivative of the function $d_{M}^{*}$, which is Theorem 2.5, we have the following corollary:

Corollary 1.2. Let $M$ be a taut complex manifold. Then $F_{M}$ is convex at $p$ if and only if

$$
\lim _{\substack{q, q^{\prime} \rightarrow p \\ q \neq q^{\prime}}} \frac{d_{M}\left(q, q^{\prime}\right)}{d_{M}^{*}\left(q, q^{\prime}\right)}=1 .
$$

The point $p \in M$ is called a Kobayashi simple point if there exists an open neighborhood $U$ of $p$ such that $d_{M}(p, q)=d_{M}^{*}(p, q)$ for all $q \in U$. For example every point of a convex domain $D$ in $\mathbb{C}^{m}$ is a Kobayashi simple point, 
because $d_{D}^{*}=d_{D}$. Corollary 1.2 implies that if $p \in M$ is the Kobayashi simple point, then $F_{M}$ is convex at $p$ (cf. [6]).

Acknowledgment. I would like to express my sincere gratitude to Professor Kiyoomi Kataoka, Professor Shoshichi Kobayashi, Professor Junjiro Noguchi, and Professor Takushiro Ochiai for helpful discussions and suggestions.

\section{Proof of Main Theorem.}

We keep the notation used in Section 1.

Definition 2.1. A holomorphic mapping $f \in \mathcal{O}(\Delta, M)$ is called an extremal mapping with respect to points $p, q \in M$ (resp. a holomorphic tangent vector $\left.\xi \in \boldsymbol{T}_{p} M\right)$, if there exists $t \in[0,1)$ such that $f(0)=p, f(t)=q$ and $d_{M}^{*}(p, q)=\rho(0, t)\left(\operatorname{resp} . F_{M}(\xi) f_{*}\left(d /\left.d \zeta\right|_{\zeta=0}\right)=\xi\right)$.

Note that in general an extremal mapping with respect to all $p, q \in M$ or $\xi \in \boldsymbol{T}_{p} M$ does not necessarily exist. In [6] M.Y. Pang showed the following theorem:

Theorem 2.2 (M.Y. Pang [6]). Let $D \subset \mathbb{C}^{m}$ be a domain containing the origin, $\left\{p_{n}\right\} \subset D$ and $\left\{q_{n}\right\} \subset D$ sequences both convergent to the origin. Suppose that $f_{n} \in \mathcal{O}(\Delta, D)$ are extremal mappings with respect to $p_{n}, q_{n} \in D$ and that they converge to $f \in \mathcal{O}(\Delta, D)$ uniformly on compact subsets. Then $f^{\prime}(0) \neq 0$ and $f$ is an extremal mapping with respect to $\left(0, f^{\prime}(0)\right)$. Moreover the following identity holds:

$$
\lim _{n \rightarrow \infty} \frac{d_{D}^{*}\left(p_{n}, q_{n}\right)}{\left\|p_{n}-q_{n}\right\|}=\frac{2 F_{D}\left(0, f^{\prime}(0)\right)}{\left\|f^{\prime}(0)\right\|},
$$

where $\|z\|=\sum_{j=1}^{m}\left|z^{j}\right|^{2}$ for all $z=\left(z^{1}, \ldots, z^{m}\right) \in \mathbb{C}^{m}$.

Let $p$ be a point of $M$. We fix an arbitrary holomorphic coordinate neighborhood $\left(U_{0}, \varphi, \Delta^{m}\right)$ about $p$ such that $\varphi(p)=0$. In fact, the following theorem holds:

Theorem 2.3. Let $\left\{p_{n}\right\} \subset M$ and $\left\{q_{n}\right\} \subset M$ be sequences both convergent to the point $p$ of $M$. Suppose that $f_{n} \in \mathcal{O}(\Delta, M)$ are extremal mappings with respect to $p_{n}, q_{n} \in M$ and that they converge to $f \in \mathcal{O}(\Delta, M)$ uniformly on compact subsets. Then $f_{*}\left(d /\left.d \zeta\right|_{\zeta=0}\right) \neq 0$ and $f$ is an extremal mapping with respect to $f_{*}\left(d /\left.d \zeta\right|_{\zeta=0}\right)$. Moreover the following identity holds:

$$
\lim _{n \rightarrow \infty} \frac{d_{M}^{*}\left(p_{n}, q_{n}\right)}{\left\|\varphi\left(p_{n}\right)-\varphi\left(q_{n}\right)\right\|}=\frac{2 F_{M}\left(f_{*}\left(d /\left.d \zeta\right|_{\zeta=0}\right)\right)}{\left\|(\varphi \circ f)_{*}\left(d /\left.d \zeta\right|_{\zeta=0}\right)\right\|} .
$$


Though the proof of the preceding theorem is the same of Theorem 2.2, we may recall the following theorem about the extension of regular holomorphic mappings, which plays an important role in the proof:

Theorem 2.4 (H.L. Royden [8]). Let $f$ be a holomorphic mapping of the unit disk $\Delta$ into an $n$-dimensional complex manifold $M$, and suppose that $f$ is regular at 0 . Then, given $r<1$, there exists a mapping $F$ of $\Delta \times \Delta^{n-1}$ into $M$, which is regular at 0 and whose restriction to $\Delta \times\{0\}$ is $f$.

From here we assume that $M$ is a taut complex manifold. Hence note that there exists an extremal mapping with respect to any $p, q \in M$ or $\xi \in \boldsymbol{T}_{p} M$.

Theorem 2.5. For any $\epsilon>0$ there exists an open neighborhood $U \subset U_{0}$ of $p$ such that

$$
\left|d_{M}^{*}\left(q, q^{\prime}\right)-2 F_{M}\left(\varphi_{*}^{-1}\left(p, \varphi(q)-\varphi\left(q^{\prime}\right)\right)\right)\right|<\epsilon\left\|\varphi(q)-\varphi\left(q^{\prime}\right)\right\|
$$

for all $q, q^{\prime} \in U$. Moreover the following identity holds:

$$
\mathcal{D} d_{M}^{*}=F_{M} .
$$

Proof. For simplicity we assume that $M$ is a domain in $\mathbb{C}^{m}$. It is sufficient to prove that for any $\epsilon>0$ there exists an open neighborhood of $p$ such that for any $q, q^{\prime} \in U$

$$
\left|d_{M}^{*}\left(q, q^{\prime}\right)-2 F_{M}\left(p, q-q^{\prime}\right)\right|<\epsilon\left\|q-q^{\prime}\right\| .
$$

Suppose the contrary; there exists a constant $\epsilon>0$ such that there are distinct points $q_{j}, q_{j}^{\prime} \in B_{\|\cdot\|}(p, 1 / j)=\left\{q \in \mathbb{C}^{m}\|q-p\|<1 / j\right\}$ for each positive integer $j$ which satisfy the following inequality:

$$
\left|d_{M}^{*}\left(q_{j}, q_{j}^{\prime}\right)-2 F_{M}\left(p, q_{j}-q_{j}^{\prime}\right)\right|>\epsilon\left\|q_{j}-q_{j}^{\prime}\right\| .
$$

Since $M$ is taut, there exists the extremal mapping $f_{j} \in \mathcal{O}(\Delta, M)$ with respect to $q_{j}, q_{j}^{\prime} \in M$ such that $f_{j}(0)=q_{j}$ and $f_{j}\left(c_{j}\right)=q_{j}^{\prime}$, where $c_{j} \in[0,1)$, for each pair of the points $q_{j}, q_{j}^{\prime} \in B_{\|\cdot\|}(0,1 / j)$. Choosing a subsequence of the sequence $\left\{f_{j}\right\}$, if necessary, we may assume that $\left\{f_{j}\right\}$ converge to $f \in$ $\mathcal{O}(\Delta, M)$ uniformly on compact subsets, and $-\left(q_{j}-q_{j}^{\prime}\right) /\left\|q_{j}-q_{j}^{\prime}\right\|$ converges to some $\xi \in \mathbb{C}^{m}$ with $\|\xi\|=1$. It follows from Theorem 2.2 that $f^{\prime}(0) \neq 0$. We easily see

$$
\begin{aligned}
\frac{f^{\prime}(0)}{\left\|f^{\prime}(0)\right\|} & =\lim _{j \rightarrow \infty} \frac{f_{j}(0)-f_{j}\left(c_{j}\right)}{0-c_{j}} \frac{\left|0-c_{j}\right|}{\left\|f_{j}(0)-f_{j}\left(c_{j}\right)\right\|} \\
& =\lim _{j \rightarrow \infty}-\frac{q_{j}-q_{j}^{\prime}}{\left\|q_{j}-q_{j}^{\prime}\right\|} \\
& =\xi .
\end{aligned}
$$


We take a sufficient large positive integer $N$ satisfying

$$
\left|\frac{d_{M}^{*}\left(q_{j}, q_{j}^{\prime}\right)}{\left\|q_{j}-q_{j}^{\prime}\right\|}-2 F_{M}\left(q^{\prime}, \xi\right)\right| \leq \frac{\epsilon}{2}
$$

and

$$
\left|2 F_{M}\left(q^{\prime}, \frac{q_{j}-q_{j}^{\prime}}{\left\|q_{j}-q_{j}^{\prime}\right\|}\right)-2 F_{M}(p, \xi)\right| \leq \frac{\epsilon}{2},
$$

for all $j>N$. Then, we have

$$
\begin{aligned}
& \left|\frac{d_{M}^{*}\left(q_{j}, q_{j}^{\prime}\right)}{\left\|q_{j}-q_{j}^{\prime}\right\|}-2 F_{M}\left(p, \frac{q_{j}-q_{j}^{\prime}}{\left\|q_{j}-q_{j}^{\prime}\right\|}\right)\right| \\
& \leq\left|\frac{d_{M}^{*}\left(q_{j}, q_{j}^{\prime}\right)}{\left\|q_{j}-q_{j}^{\prime}\right\|}-2 F_{M}\left(q^{\prime}, \xi\right)\right| \\
& \quad+\left|2 F_{M}\left(q^{\prime}, \xi\right)-2 F_{M}\left(p, \frac{q_{j}-q_{j}^{\prime}}{\left\|q_{j}-q_{j}^{\prime}\right\|}\right)\right| \\
& \leq \epsilon .
\end{aligned}
$$

This is a contradiction. Thus we finish the proof of the first part of this theorem.

We fix a Hermitian metric $h$ on $M$. Then the following facts are wellknown:

$$
\begin{aligned}
\lim _{\substack{u \rightarrow v \\
t \rightarrow 0}} \frac{\varphi(\exp t u)-\varphi(q)}{t} & =\xi ; \\
\lim _{\substack{u \rightarrow v \\
t \rightarrow 0}} \frac{\varphi(\exp t u)-\varphi(q)}{\|\varphi(\exp t u)-\varphi(q)\|} & =\frac{\xi}{\left\|\varphi_{*}(\xi)\right\|},
\end{aligned}
$$

where $\xi \in \boldsymbol{T}_{p} M$ with $v=\xi+\bar{\xi}$ and $u \in T_{q} M$. It follows from the first part of this theorem and the preceding facts that

$$
\begin{aligned}
\mathcal{D} d_{M}^{*}(v) & =\lim _{\substack{u \rightarrow v \\
t \rightarrow 0}} \frac{d_{M}^{*}(q, \exp t u)}{|t|} \\
& =\lim _{\substack{u \rightarrow v \\
t \rightarrow 0}} \frac{d_{M}^{*}(q, \exp t u)}{\|\varphi(\exp t u)-\varphi(q)\|} \frac{\|\varphi(\exp t u)-\varphi(q)\|}{|t|} \\
& =2 F_{M}\left(\xi /\left\|\varphi_{*}(\xi)\right\|\right)\left\|\varphi_{*}(\xi)\right\| \\
& =F_{M}(v) .
\end{aligned}
$$

Thereby we conclude the whole proof. 
Lemma 2.6 (H.L. Royden [7]). Let $h$ be a Hermitian metric on $M$ and $p$ a point of $M$. Then, there exists a constant $L^{\prime}>0$ such that for any $\xi \in \boldsymbol{T}_{p} M$

$$
L^{\prime}\|\xi\|_{h}<F_{M}(\xi)
$$

where $\|\xi\|_{h}$ is the length of $\xi$ induced by $h$.

Theorem 2.7 (S. Kobayashi [3]). For any $\xi \in \boldsymbol{T}_{p} M$ there exist $n$ holomorphic tangent vectors $\xi_{1}, \ldots, \xi_{n} \in \boldsymbol{T}_{p} M(n \leq 2 m)$ satisfying the following:

(i) $\xi_{1} \ldots, \xi_{n}$ are linearly independent over $\mathbb{R}$;

(ii) $\xi=\sum_{j=1}^{n} \xi_{j}$;

(iii) $\hat{F}_{M}(\xi)=\sum_{j=1}^{n} F_{M}\left(\xi_{j}\right)$.

Lemma 2.8. Let $p$ be a point of $M$. Then, there exists a constant $L>0$ satisfying the following: For any $\xi \in \boldsymbol{T}_{p} M$ we take $\xi_{1}, \ldots, \xi_{n} \in \boldsymbol{T}_{p} M$ as in the preceding theorem. Then the following inequality holds:

$$
\|\xi\|_{h} \leq L \sum_{j=1}^{n}\left\|\xi_{j}\right\|_{h}
$$

Proof. Since $\hat{F}_{M}$ is continuous, there exists a constant $L^{\prime \prime}>0$ such that

$$
L^{\prime \prime}\|\xi\|_{h} \geq \hat{F}_{M}(\xi)
$$

for all $\xi \in \boldsymbol{T}_{p} M$. We take $\xi_{1}, \ldots, \xi_{n} \in \boldsymbol{T}_{p} M$ as in Theorem 2.7. By Lemma 2.6 we see

$$
\sum_{j=1}^{n} F_{M}\left(\xi_{j}\right) \geq L^{\prime} \sum_{j=1}^{n}\left\|\xi_{j}\right\|_{h} .
$$

Because of the preceding two inequalities, we have

$$
L^{\prime \prime}\|\xi\|_{h} \geq \hat{F}_{M}(\xi)=\sum_{j=1}^{n} F_{M}\left(\xi_{j}\right) \geq L^{\prime} \sum_{j=1}^{n}\left\|\xi_{j}\right\|_{h} .
$$

Thereby the proof is concluded.

We recall the following fact:

Remark 2.9. We fix a positive integer $l$. Take any two points $q, q^{\prime}$ of $M$ such that $d_{M}^{(l)}\left(q, q^{\prime}\right)<\infty$. Because $M$ is taut, there exist $l+1$ points $q_{1}=$ $q, q_{2}, \ldots, q_{l}, q_{l+1}=q^{\prime} \in M$ such that $d_{M}^{(l)}\left(q, q^{\prime}\right)=\sum_{j=1}^{l} d_{M}^{*}\left(q_{j}, q_{j+1}\right)$.

Lemma 2.10. For any open neighborhood $W \subset U_{0}$ of $p$ and positive integer $l$, there exists an open neighborhood $V \subset W$ of $p$ satisfying the following:

For any $q, q^{\prime} \in V$, we take the $l+1$ points $q_{1}, \ldots, q_{l+1}$ as in Remark 2.9. Then, $q_{1}, \ldots, q_{l+1}$ are contained in $W$. 
Proof. Since $M$ is taut, $M$ is hyperbolic (i.e., $d_{M}$ is distance and the topology induced by it is the same of $M)$. Choosing a constant $R>0$, we may assume that $W=B_{d_{M}}(p, R)=\left\{q \in M \mid d_{M}(p, q)<R\right\}$. There exists a constant $r>$ 0 satisfying $\varphi^{-1}\left(B_{\|\cdot\|}(0, r)\right) \subset W$, where $B_{\|\cdot\|}(0, r)=\left\{z \in \mathbb{C}^{m} \mid\|z\|<r\right\}$. Set $V=\varphi^{-1}\left(B_{d_{B_{\|\cdot\|}(0, r)}}(0, R / 4)\right)$. For any two points $q, q^{\prime} \in V$, there exist $l+1$ points $q_{1}, \ldots, q_{l+1} \in M$ as in Remark 2.9. Then, for any $q_{j}$ it follows that

$$
\begin{aligned}
d_{M}\left(p, q_{j}\right) & \leq d_{M}(p, q)+d_{M}\left(q, q_{j}\right) \\
& \leq d_{M}(p, q)+d_{M}^{(j)}\left(q, q_{j}\right) \\
& \leq d_{M}(p, q)+d_{M}^{(l)}\left(q, q^{\prime}\right) .
\end{aligned}
$$

Because the Kobayashi distance has the distance-decreasing property and $d_{B_{\|\cdot\|}(l)}^{(l)}(0, r)=d_{B_{\|\cdot\|}(0, r)}$, we have

$$
\begin{aligned}
d_{M}\left(p, q_{j}\right) \leq & d_{B_{\|\cdot\|}(0, r)}(\varphi(p), \varphi(q))+d_{B_{\|\cdot\|}(0, r)}\left(\varphi(q), \varphi\left(q^{\prime}\right)\right) \\
\leq & d_{B_{\|\cdot\|}(0, r)}(\varphi(p), \varphi(q)) \\
& +d_{B_{\|\cdot\|}(0, r)}(\varphi(q), \varphi(p))+d_{B_{\|\cdot\|}(0, r)}\left(\varphi(p), \varphi\left(q^{\prime}\right)\right) \\
\leq & (3 / 4) R .
\end{aligned}
$$

Thus $q_{j}$ is contained in $W$. The proof is thereby concluded.

Lemma 2.11. There exist an open neighborhood $V$ of $p$ and a constant $C>0$ such that for any points $q, q^{\prime} \in V$ and positive integer $l$,

$$
\sum_{j=1}^{l}\left\|\varphi\left(q_{j}\right)-\varphi\left(q_{j+1}\right)\right\| \leq C\left\|\varphi(q)-\varphi\left(q^{\prime}\right)\right\|,
$$

where $q_{1}, \ldots, q_{l+1}$ are points of $M$ chosen as in Remark 2.9 .

Proof. Since $M$ is taut, $F_{M}$ is continuous. The Kobayashi distance $d_{M}$ is an integrated form of $F_{M}$, therefore there exist an open neighborhood $W$ of $p$ and a constant $C^{\prime}>0$ such that

$$
C^{\prime}\left\|\varphi(q)-\varphi\left(q^{\prime}\right)\right\| \leq d_{M}\left(q, q^{\prime}\right)
$$

for any $q, q^{\prime} \in W$. We choose a sufficiently small open neighborhood $V \subset W$ of $p$ as in the preceding lemma. For any $q, q^{\prime} \in V$ we take $l+1$ points $q_{1}, \ldots, q_{l+1} \in W$ as in Remark 2.9. Then, we have

$$
\begin{aligned}
& C^{\prime} \sum_{j=1}^{l}\left\|\varphi\left(q_{j}\right)-\varphi\left(q_{j+1}\right)\right\| \leq \sum_{j=1}^{l} d_{M}\left(q_{j}, q_{j+1}\right) \\
& \leq \sum_{j=1}^{l} d_{M}^{*}\left(q_{j}, q_{j+1}\right)=d_{M}^{(l)}\left(q, q^{\prime}\right) \leq d_{M}^{*}\left(q, q^{\prime}\right) .
\end{aligned}
$$


On the other hand, choosing a small constant $R>0$, we may assume that $V=\varphi^{-1}\left(B_{\|\cdot\|}(0, R)\right)$ and $\varphi^{-1}\left(B_{\|\cdot\|}(0,2 R)\right) \subset W$. Then, there exists a constant $C^{\prime \prime}$ such that

$$
d_{B_{\|\cdot\|}(0,2 R)}\left(\varphi(q), \varphi\left(q^{\prime}\right)\right) \leq C^{\prime \prime}\left\|\varphi(q)-\varphi\left(q^{\prime}\right)\right\|
$$

for any $q, q^{\prime} \in V$. Thus, we have

$$
d_{M}^{*}\left(q, q^{\prime}\right) \leq d_{B_{\|\cdot\|}(0,2 R)}\left(\varphi(q), \varphi\left(q^{\prime}\right)\right) \leq C^{\prime \prime}\left\|\varphi(q)-\varphi\left(q^{\prime}\right)\right\| .
$$

Combining (2.23) and (2.25), we have

$$
C^{\prime} \sum_{j=1}^{l}\left\|\varphi\left(q_{j}\right)-\varphi\left(q_{j+1}\right)\right\| \leq C^{\prime \prime}\left\|\varphi(q)-\varphi\left(q^{\prime}\right)\right\| .
$$

Thereby we conclude the proof.

The next lemma is a key to proving the Main Theorem.

Lemma 2.12. For any $\epsilon>0$ and positive integer $l \geq 2 m$, there exists an open neighborhood $U \subset U_{0}$ of $p$ such that

$$
\left|d_{M}^{(l)}\left(q, q^{\prime}\right)-2 \hat{F}_{M}\left(\varphi_{*}^{-1}\left(p, \varphi(q)-\varphi\left(q^{\prime}\right)\right)\right)\right|<\epsilon\left\|\varphi(q)-\varphi\left(q^{\prime}\right)\right\|
$$

for all $q, q^{\prime} \in U$.

Proof. For simplicity we assume that $M$ is a domain in $\mathbb{C}^{m}$. We take the distinct two points $q, q^{\prime} \in U_{0}$ arbitrarily. We can choose the points $q_{1}=q, q_{2}, \ldots, q_{l}, q_{l+1}=q^{\prime} \in M$ as in Remark 2.9, and $n$ holomorphic tangent vectors $\left(p, \xi_{1}\right), \ldots,\left(p, \xi_{n}\right) \in M \times \mathbb{C}^{m}$ for $\left(p, q-q^{\prime}\right) \in M \times \mathbb{C}^{m}$ as in Theorem 2.7, where $n \leq 2 m$. Clearly it follows that

$$
\sum_{j=1}^{l} d_{M}^{*}\left(q_{j}, q_{j+1}\right)=d_{M}^{(l)}\left(q, q^{\prime}\right) \leq \sum_{j=1}^{n} d_{M}^{*}\left(q+\sum_{k=0}^{j-1} \xi_{k}, q+\sum_{k=1}^{j} \xi_{k}\right),
$$

where $\xi_{0}=0$. Thus we have

$$
\begin{aligned}
\sum_{j=1}^{l} \frac{d_{M}^{*}\left(q_{j}, q_{j+1}\right)}{\left\|q-q^{\prime}\right\|} & =\frac{d_{M}^{(l)}\left(q, q^{\prime}\right)}{\left\|q-q^{\prime}\right\|} \\
& \leq \sum_{j=1}^{n} \frac{d_{M}^{*}\left(q+\sum_{k=0}^{j-1} \xi_{k}, q+\sum_{k=1}^{j} \xi_{k}\right)}{\left\|\xi_{j}\right\|} \frac{\left\|\xi_{j}\right\|}{\left\|q-q^{\prime}\right\|}
\end{aligned}
$$


We easily see that

$$
\begin{aligned}
& \sum_{j=1}^{l} \frac{d_{M}^{*}\left(q_{j}, q_{j+1}\right)}{\left\|q_{j+1}-q_{j}\right\|} \frac{\left\|q_{j+1}-q_{j}\right\|}{\left\|q-q^{\prime}\right\|} \\
& =\frac{d_{M}^{(l)}\left(q, q^{\prime}\right)}{\left\|q-q^{\prime}\right\|} \leq \sum_{j=1}^{n} \frac{d_{M}^{*}\left(q+\sum_{k=0}^{j-1} \xi_{k}, q+\sum_{k=1}^{j} \xi_{k}\right)}{\left\|\xi_{j}\right\|} \frac{\left\|\xi_{j}\right\|}{\left\|q-q^{\prime}\right\|} .
\end{aligned}
$$

We arbitrarily fix $\epsilon>0$. Then we take an open neighborhood $U$ of $p$ as in Theorem 2.5. Then for any $q, q^{\prime} \in U$ we have

$$
\begin{aligned}
& \sum_{j=1}^{l}\left(2 F_{M}\left(p, \frac{q_{j+1}-q_{j}}{\left\|q_{j+1}-q_{j}\right\|}\right)-\epsilon\right) \frac{\left\|q_{j+1}-q_{j}\right\|}{\left\|q-q^{\prime}\right\|} \\
& \leq \frac{d_{M}^{(l)}\left(q, q^{\prime}\right)}{\left\|q-q^{\prime}\right\|} \leq \sum_{j=1}^{n}\left(2 F_{D}\left(p, \frac{\xi_{j}}{\left\|\xi_{j}\right\|}\right)+\epsilon\right) \frac{\left\|\xi_{j}\right\|}{\left\|q-q^{\prime}\right\|} .
\end{aligned}
$$

Replacing $U$ by a smaller one, we may assume that the conditions in Lemmas 2.8 and 2.11 hold. Thus, we have

$$
\begin{aligned}
& 2 \sum_{j=1}^{l}\left(F_{M}\left(p, \frac{q_{j+1}-q_{j}}{\left\|q-q^{\prime}\right\|}\right)\right)-C \epsilon \\
& \leq \frac{d_{M}^{(l)}\left(q, q^{\prime}\right)}{\left\|q-q^{\prime}\right\|} \leq 2 \sum_{j=1}^{n}\left(F_{D}\left(p, \frac{\xi_{j}}{\left\|q-q^{\prime}\right\|}\right)\right)+L \epsilon .
\end{aligned}
$$

Because

$$
\begin{aligned}
\sum_{j=1}^{l} F_{M}\left(p, \frac{q_{j+1}-q_{j}}{\left\|q-q^{\prime}\right\|}\right) & \geq \sum_{j=1}^{l} \hat{F}_{M}\left(p, \frac{q_{j+1}-q_{j}}{\left\|q-q^{\prime}\right\|}\right) \\
& \geq \hat{F}_{M}\left(p, \sum_{j=1}^{l} \frac{q_{j+1}-q_{j}}{\left\|q-q^{\prime}\right\|}\right) \\
& =\hat{F}_{M}\left(p, \frac{q-q}{\left\|q-q^{\prime}\right\|}\right)
\end{aligned}
$$

and

$$
\begin{aligned}
\sum_{j=1}^{n} F_{M}\left(p, \frac{\xi_{j}}{\left\|\xi_{j}\right\|}\right) & =\hat{F}_{M}\left(p, \frac{q^{\prime}-q}{\left\|q^{\prime}-q\right\|}\right) \\
& =\hat{F}_{M}\left(p, \frac{q^{\prime}-q}{\left\|q^{\prime}-q\right\|}\right)
\end{aligned}
$$


we obtain

$$
\begin{aligned}
& 2 \hat{F}_{M}\left(p, \frac{q-q^{\prime}}{\left\|q-q^{\prime}\right\|}\right)-C \epsilon \\
& \leq \frac{d_{M}^{(l)}\left(q, q^{\prime}\right)}{\left\|q-q^{\prime}\right\|} \leq 2 \hat{F}_{M}\left(p, \frac{q-q^{\prime}}{\left\|q-q^{\prime}\right\|}\right)+L \epsilon .
\end{aligned}
$$

The constants $C$ and $L$ are independent of $l$. Hence the proof is finished.

Proof of Main Theorem 1.1. We take any Hermitian metric $h$ on $M$. It follows from the preceding lemma that

$$
\lim _{t \rightarrow 0} \frac{d_{M}(\exp t v, q)}{\|\varphi(\exp t v)-\varphi(q)\|}=\hat{F}_{M}\left(\frac{\xi}{\left\|\varphi_{*}(\xi)\right\|}\right),
$$

where $\xi \in \boldsymbol{T}_{p} M$ with $v=\xi+\bar{\xi}$. Hence we have

$$
\begin{aligned}
\mathcal{D} d_{M}(v) & =\lim _{\substack{u \rightarrow 0 \\
t \rightarrow 0}} \frac{d_{M}(q, \exp t u)}{|t|} \\
& =\lim _{\substack{u \rightarrow v \\
t \rightarrow 0}} \frac{d_{M}(q, \exp t u)}{\|\varphi(\exp t u)-\varphi(q)\|} \frac{\|\varphi(\exp t u)-\varphi(q)\|}{|t|} \\
& =2 \hat{F}_{M}\left(\frac{\xi}{\left\|\varphi_{*}(\xi)\right\|}\right)\left\|\varphi_{*}(\xi)\right\| \\
& =2 \hat{F}_{M}(\xi) \\
& =\hat{F}_{M}(v) .
\end{aligned}
$$

Thereby the proof is completed.

It is easy to see Corollary 1.2 by Main Theorem 1.1 and Theorem 2.5.

\section{References}

[1] M. Jarnicki and P. Pflug, Invariant Distances and Metrics in Complex Analysis, Walter de Gruyter Expositions in Mathematics, 9, Walter de Gruyter \& Co., Berlin, 1993.

[2] S. Kobayashi, Intrinsic distances, measures and geometric function theory, Bull. Amer. Math. Soc., 82(3) (1976), 357-416.

[3] _ _ A new invariant infinitesimal metric, Internat. J. Math., 1(1) (1990), 83-90.

[4] L. Lempert, La métrique de Kobayashi et la représentation des domaines sur la boule, Bull. Soc. Math. France, 109(4) (1981), 427-474.

[5] J. Noguchi and T. Ochiai, Geometric Function Theory in Several Complex Variables, Translated from the Japanese by Noguchi; Translations of Mathematical Monographs, 80, American Mathematical Society, Providence, RI, 1990.

[6] M.Y. Pang, On infinitesimal behavior of the Kobayashi distance, Pacific J. Math., 162(1) (1994), 121-141. 
[7] H.L. Royden, Remarks on the Kobayashi metric, "Several complex variables II", pp. 125-137, Lecture Notes in Math., Vol. 185, Springer, Berlin, 1971.

[8] _ The Extension of regular holomorphic maps, Proc. Amer. Math. Soc., 43(2) (1974), 306-310.

Received November 20, 1997 and revised April 10, 1999.

Graduate School of Mathematical Sciences

UNIVERSITY OF TOKYO

8-1 Komaba 3-Chome Meguro-Ku

TOKYO

JAPAN

E-mail address: masashi@ms.u-tokyo.ac.jp 